\begin{tabular}{|c|c|}
\hline Title & Interplay between magnetism and conductivity in the one dimensional organic conductor $\operatorname{TPP}[\mathrm{Fe}(\mathrm{Pc})(\mathrm{CN}) 2] 2$ \\
\hline Author(s) & Kimata, M.; Takahide, Y.; Harada, A .; Satsukawa, H.; Hazama, K.; Terashima, T.; Uji, S.; Naito, T.; Inabe, T. \\
\hline Citation & $\begin{array}{l}\text { Physical Review B, 80(8), } 085110 \\
\text { https://doi.org/10.1103/PhysRevB.80.085110 }\end{array}$ \\
\hline Issue Date & $2009-08$ \\
\hline Doc URL & http:/hdl. handle.net/2115/39403 \\
\hline Rights & ๑2009 The A merican Physical Society \\
\hline Type & article \\
\hline File Information & PRB80-8_085110.pdf \\
\hline
\end{tabular}

Instructions for use 


\title{
Interplay between magnetism and conductivity in the one-dimensional organic conductor $\mathrm{TPP}\left[\mathrm{Fe}(\mathrm{Pc})(\mathrm{CN})_{2}\right]_{2}$
}

\author{
M. Kimata, ${ }^{*}$ Y. Takahide, A. Harada, H. Satsukawa, K. Hazama, ${ }^{\dagger}$ T. Terashima, and S. Uji ${ }^{\dagger}$ \\ National Institute for Materials Science (NIMS), Tsukuba 305-0003, Japan \\ T. Naito and T. Inabe \\ Division of Chemistry, Graduate School of Science, Hokkaido University, Sapporo 060-0810, Japan \\ (Received 16 October 2008; revised manuscript received 24 April 2009; published 18 August 2009)
}

\begin{abstract}
The strongly nonlinear current-voltage $(I-V)$ characteristics and the large negative magnetoresistance (MR) are observed at low temperatures in the one-dimensional organic conductor TPP $\left[\mathrm{Fe}(\mathrm{Pc})(\mathrm{CN})_{2}\right]_{2}$. The nonlinear $I-V$ curves are interpreted as the transport of the electrons and holes excited from the charge ordered state on the $\mathrm{Fe}(\mathrm{Pc})(\mathrm{CN})_{2}$ chains, whose energy gap strongly depends on electric fields. The negative MR is enhanced as temperature decreases, and the resistance steeply changes over four orders of magnitude around $15 \mathrm{~T}$ at 1.5 $\mathrm{K}$. This decrease is associated with a magnetic torque anomaly, and the energy gap is rapidly reduced around $15 \mathrm{~T}$. The detailed analyses of the $I-V$ curves show that the electric flux distribution, i.e., the dimensionality of the Coulomb interaction between the carriers, also changes around $15 \mathrm{~T}$.
\end{abstract}

DOI: $10.1103 /$ PhysRevB.80.085110

PACS number(s): 72.20.Ht, 71.27.+a, 72.80.Le, 75.47. $-\mathrm{m}$

\section{INTRODUCTION}

Interplay between magnetism and conductivity has brought us various intriguing phenomena especially in strongly correlated materials. ${ }^{1-10}$ The large magnetoresistance (MR) effect arising from such interplay, for instance, is still a central issue in solid-state physics. Some perovskite manganites show quite large negative MR, which is known as the colossal MR (CMR). ${ }^{2-4}$ However, except for the manganites, such large MR effect, which amounts to several orders of magnitude, is rarely observed.

Magnetic organic conductors have attracted much attention due to their unique physical properties recently., ${ }^{5,10}$ Among them, the Fe-dicyanophthalocyanine conductor $\mathrm{TPP}\left[\mathrm{Fe}(\mathrm{Pc})(\mathrm{CN})_{2}\right]_{2}$ is quite interesting. ${ }^{8}$ The resistance gradually decreases by two orders of magnitude up to $40 \mathrm{~T}$ for $T=20 \mathrm{~K}$ when the magnetic field is applied parallel to the $a$ axis. In spite of the intensive studies, the origin has not been clarified yet. ${ }^{7,8,11}$ TPP $\left[\mathrm{Fe}(\mathrm{Pc})(\mathrm{CN})_{2}\right]_{2}$ has one-dimensional (1D) conducting chains of stacked dicyano(phthalocyaninato)iron $\mathrm{Fe}(\mathrm{Pc})(\mathrm{CN})_{2}$ molecules which are parallel to the $c$ axis of the crystal (TPP=tetraphenylphosphonium, $\mathrm{Pc}$ $=$ phthalocyanine $).{ }^{12,13}$ The $\mathrm{Fe}(\mathrm{Pc})(\mathrm{CN})_{2}$ molecule has both $\pi$ electrons on the Pc ligand and the local $3 d$ magnetic moment at the central $\mathrm{Fe}^{3+}$ ion, so that the conduction electrons are strongly coupled with the local moments: a large $\pi-d$ interaction is present. Because of unquenching of the $3 d$ orbital angular momentum, the Fe local moment is quite anisotropic (the easy axis is nearly parallel to the $a$ axis). ${ }^{14} \mathrm{Al}-$ though the conduction band is partially occupied formally (3/4 filling), the temperature dependence of the electrical resistivity shows semiconducting behavior from room temperature to the lowest temperature with no lattice distortion. In this material, the energy gap between the highestoccupied-molecular-orbital (HOMO) and the lowest-unoccupied-molecular-orbital (LUMO) is expected to be about 5 $\mathrm{eV} \cdot{ }^{14}$ Note that the experimentally reported activation energy gap at low temperatures is much smaller than the HOMO-
LUMO gap. This fact shows that the energy gap originates from strongly correlated effects (i.e., Coulomb repulsion): the ground state is a 1D charge order. ${ }^{13,15}$

In this paper, we report systematic measurements of MR, current-voltage $(I-V)$ characteristics, and magnetic torque of $\mathrm{TPP}\left[\mathrm{Fe}(\mathrm{Pc})(\mathrm{CN})_{2}\right]_{2}$. The change in the MR reaches four orders of magnitude below $10 \mathrm{~K}$. We show that the quite large negative MR is caused by the magnetic-field-dependent energy gap of the charge ordered state, which depends on the magnetic state of the $3 d$ moments in the $1 \mathrm{D} \mathrm{Fe}(\mathrm{Pc})(\mathrm{CN})_{2}$ chains. Moreover, we find that the functional form of the Coulomb interaction between carriers also depends on the magnetic state.

\section{EXPERIMENTAL}

Needlelike single-crystal samples with typical dimensions of $100 \mu \mathrm{m} \times 100 \mu \mathrm{m} \times 1 \mathrm{~mm}$ were synthesized by a standard electrochemical method. ${ }^{13}$ Resistance measurements were performed by a two-terminal method with current along the $c$ axis (longest direction). The results were identical to those for a four-terminal method in the current range for $I$ $>10^{-10} \mathrm{~A}$ : the contact resistance is negligible. The typical electrodes spacing is about $40 \mu \mathrm{m}$ for the present sample. The magnetic torque measurements were performed by commercial microcantilevers. ${ }^{16}$

\section{RESULTS AND DISCUSSIONS}

The temperature dependence of the low-bias conductance at 0 and $17.8 \mathrm{~T}$ is shown in Fig. 1. Magnetic field is applied to the $a$ axis of the crystal, and the bias voltage is kept at $\pm 10 \mathrm{mV}$, where the Ohmic conduction is confirmed. Below $30 \mathrm{~K}$, the conductance follows the Arrhenius law $\sigma(T)$ $=\sigma_{0} \exp \left(-\Delta_{0} / 2 k_{B} T\right)$. The saturation behavior of the conductance at low temperatures is due to the limitation of the measurement. The activation energy $\Delta_{0} / k_{B}$ at $0 \mathrm{~T}$ is estimated as $650 \mathrm{~K}$, where $k_{B}$ is the Boltzmann constant. This value is 


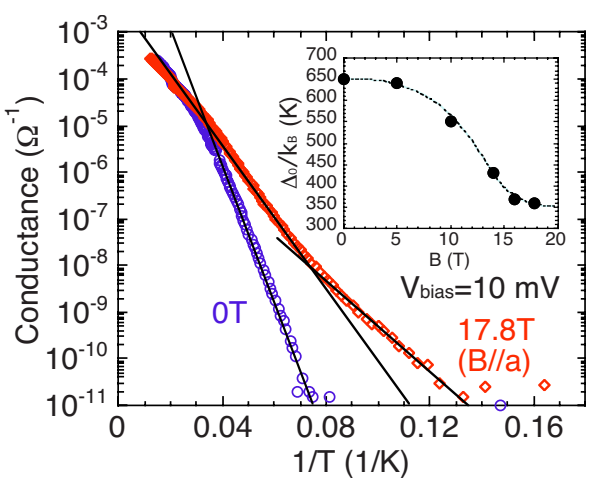

FIG. 1. (Color online) Temperature dependence of the low-bias conductance at $0(\bigcirc)$ and $17.8 \mathrm{~T}(\diamond)$. Inset shows the magnetic field dependence of the activation energy for $T=30-13 \mathrm{~K}$. The broken line is a guide for the eye.

slightly larger than the previously reported one, $\Delta_{0} / k_{B}$ $\approx 600 \mathrm{~K} .{ }^{8}$ At $17.8 \mathrm{~T}$, the conductance is enhanced (negative $\mathrm{MR}$ ), and consequently, the activation energy is significantly reduced. We note that the activation energy changes around $12.5 \mathrm{~K}$, from $\Delta_{0} / k_{B}=360 \mathrm{~K}$ for $T=30-13 \mathrm{~K}$ to $180 \mathrm{~K}$ for $T<12 \mathrm{~K}$. The origin is not clear at the moment. An important feature is that the activation energy for $T=30-13 \mathrm{~K}$ gradually decreases with field and has a tendency to saturate at $15 \mathrm{~T}$ (inset of Fig. 1).

The temperature dependence of the $I-V$ curves at $0 \mathrm{~T}$ is shown in Fig. 2(a). The nonlinear $I-V$ characteristics are well visible below $13 \mathrm{~K}$. Charge ordered states have been well understood in the framework of extended Hubbard models, ${ }^{17}$ in which three parameters, on-site Coulomb interaction $(u)$, intersite Coulomb interaction $(v)$, and transfer integral between the neighboring sites $(t)$ are involved. When $u$ and $v$ are much larger than $t$, the ground states become charge ordered insulators even for quarter-filled systems, as in the case of this material. The energy gap is generally a function of these three parameters. In the charge ordered systems, the carriers are thermally excited electrons and holes. The nonlinear $I-V$ characteristics have been understood in terms of electric field dependence of the energy-gap $\Delta(E) .{ }^{18,19}$ Thus, $\sigma(E, T)=\sigma_{0} \exp \left(-\Delta(E) / 2 k_{B} T\right)$. In the present case, the electron-hole pairs likely lie in the same chains (essentially $1 \mathrm{D}$ system). If the electric fluxes between the excited electron and hole are completely confined in the $\mathrm{Fe}(\mathrm{Pc})(\mathrm{CN})_{2}$ chain (the dielectric constant is predominant along the chain), the Coulomb potential, corresponding to the binding energy between the electron and hole, has a form, $\phi(r)$ $=U_{0}|r|$. Here, $r$ is the distance between them and $U_{0}$ is a free parameter, depending on the dielectric constant of the chains. We introduce a cutoff length $\lambda$, where the 1D potential (i.e., linear potential) can be assumed. This cutoff length is given by $d \sqrt{\epsilon_{c} / \epsilon_{i}}$ on the assumption of $\epsilon_{c} \gg \epsilon_{i}$, where $d$ is the diameter of the conducting chain, and $\epsilon_{c}$ and $\epsilon_{i}$ are the dielectric constant of the conducting chain and the other part surrounding the chain, respectively. ${ }^{20}$ In the range extended over $\lambda$, the potential is proportional to $1 / r$ [i.e., threedimensional (3D) Coulomb potential], which approaches to a constant value at infinity. Here, we assume a flat potential in the range of $r>\lambda$ for simplicity. The schematic shape of the
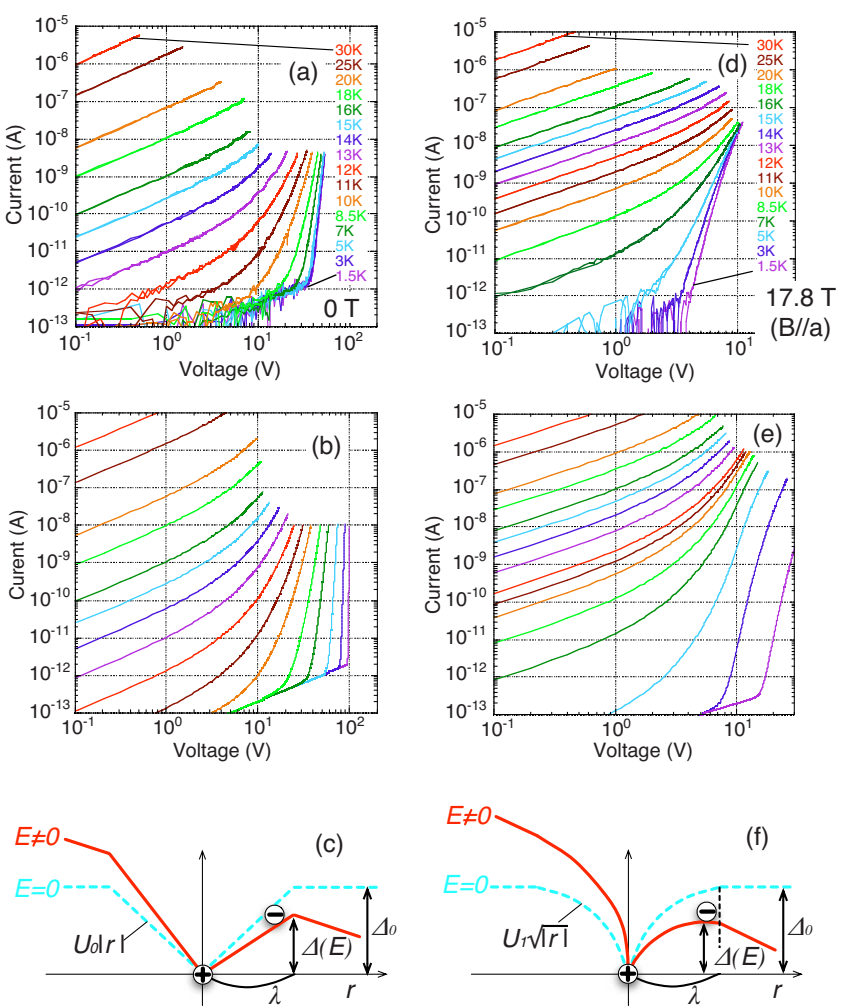

FIG. 2. (Color online) $I-V$ curves at (a) 0 and (d) 17.8 T. Simulated curves with assumptions of (b) the linear potential (1D case), and (e) the square-root potential. Schematic pictures of the Coulomb potentials used for the simulations; (c) the linear potential and (f) the square-root potential. See text for the details.

potential will be given by the dotted line in Fig. 2(c). The electric field dependence of the energy gap is given by $\Delta(E)=\Delta_{0}-e E \lambda$ as shown by the solid line. The final formula of the conductance would be

$$
\sigma(E, T)=\sigma_{0} \exp \left(-\left(\Delta_{0}-e E \lambda\right) / 2 k_{B} T\right)+\sigma_{1} .
$$

The $I-V$ curves simulated by Eq. (1) are shown in Fig. 2(b). We take $\lambda=20 \mathrm{~nm}$ and $\sigma_{1}=2 \times 10^{-14} \Omega^{-1}$ for the simulation. $\Delta_{0} / k_{B}$ is set on $650 \mathrm{~K}$. Here, we add the electric-fieldindependent part of the conductance $\sigma_{1}$ to reproduce the small Ohmic tails. The Ohmic tails have sample or electrode dependence, so that its origin may be a tiny leakage current due to some insulation failure. The other parts are sample independent, showing the intrinsic nature. We also simulated the $I-V$ curves using a 3D potential $[\phi(r) \propto 1 / r]$ for $r>\lambda$, but there is no significant difference between two cases (flat and 3D potentials for $r>\lambda$ ). Therefore, a flat potential for $r>\lambda$ is reasonable assumption and gives no significant change in our conclusion. The $I-V$ curves simply shift to lower electric field region as the electrode spacing shortens. The fact means that the effect of the electric field inhomogeneity near the contacts on the nonlinear $I-V$ curves is negligibly small. Figure $2(\mathrm{~d})$ shows the $I-V$ curves at $17.8 \mathrm{~T}(B \| a)$. The $I-V$ curves shift to lower electric field region due to the negative MR. The most important feature is the negative curvature [i.e., $d^{2} \log (I) / d \log (V)^{2}<0$ ] of the $I-V$ curves in the strongly nonlinear region below $5 \mathrm{~K}$ and above $5 \mathrm{~V}$. Note 


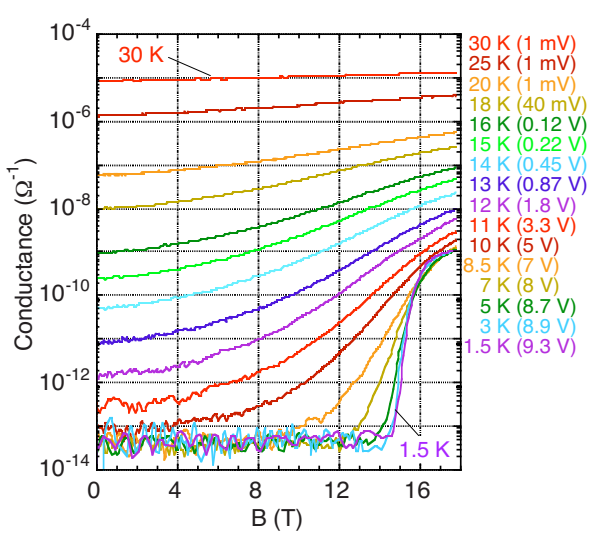

FIG. 3. (Color online) Magnetic field dependence of the conductance with constant voltage at various temperatures. The measurement temperatures and the bias voltages are shown outside the graph.

that the positive curvature is always observed in the whole region at $0 \mathrm{~T}$. This fact indicates that the $1 \mathrm{D}$ Coulomb potential model is not appropriate at $17.8 \mathrm{~T}$. If the Coulomb potential of $\phi(r)=U_{1} \sqrt{|r|}$ is assumed, the negative curvature is well reproduced [Fig. 2(e)]. We also introduce the cutoff length $\lambda=30 \mathrm{~nm}$. In this case, the energy gap is given by $\Delta(E)=\Delta_{0}-e \underline{E} \lambda$ for $E<U_{1} /(2 e \sqrt{\lambda})$ and $\Delta(E)=U_{1}^{2} /(4 e E)$ for $E \geq U_{1} /(2 e \sqrt{\lambda})$. The parameter $\Delta_{0} / k_{B}$ is set on $360 \mathrm{~K}$ for $T$ $=30-13 \mathrm{~K}$, and $180 \mathrm{~K}$ for $T=12-1.5 \mathrm{~K}$. The simulations are in good agreement with the experimental results: the above potential forms well explain the experimental results. Deviation from the simulation seen at low temperatures might be explained by quantum tunneling. The similar deviation is observed in two-dimensional (2D) charge ordered systems. ${ }^{19}$

The magnetic field dependence of the conductance at constant bias voltage is shown in Fig. 3. The bias voltage is chosen so that the current remains around $10 \mathrm{nA}$ for $B$ $=17.8 \mathrm{~T}$. As the temperature decreases, the positive magnetoconductance (negative MR) is enhanced, and the change in the conductance amounts to four orders of magnitude or over below $10 \mathrm{~K}$. Moreover, a steep increase in the conductance around $15 \mathrm{~T}$ is observed for $T=1.5 \mathrm{~K}$. The present result suggests that the energy gap is reduced rapidly around $15 \mathrm{~T}$. These results are consistent with the magnetic field dependence of the energy gap (see inset of Fig. 1). This large negative MR can be ascribed to the large $\pi$ - $d$ interaction because the isomorphic compound $\mathrm{TPP}\left[\mathrm{Co}(\mathrm{Pc})(\mathrm{CN})_{2}\right]_{2}$, which has no local magnetic moment, does not show negative MR. ${ }^{15}$

The magnetic field dependence of the magnetic torque for $B \| a$ is shown in Fig. 4(a). At $1.5 \mathrm{~K}$, the torque also changes rapidly around $15 \mathrm{~T}$, which is evident in the derivative curve as shown in the inset of Fig. 4(a). This anomaly suggests the existence of a change in the magnetic state around $15 \mathrm{~T}$. As temperature increases, the anomaly becomes gradually less evident, and not appreciable above $13 \mathrm{~K}$.

The stability of charge ordered states and the energy gap strongly depend on the transfer integral $(t)$ and the Coulomb correlation, which is well established in the $\theta$ phase BEDTTTF salts. ${ }^{21}$ In the present case, the mechanism of the
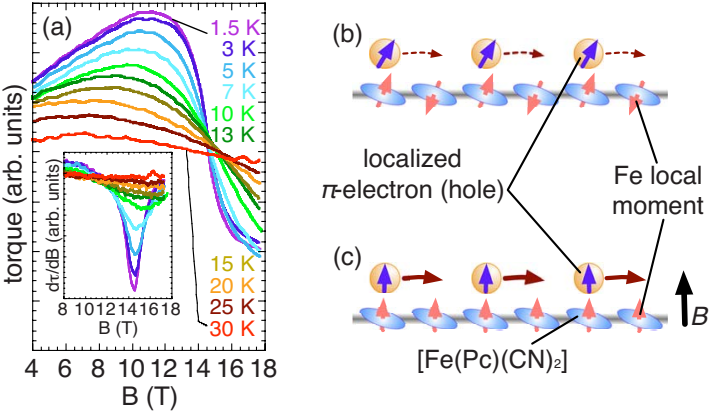

FIG. 4. (Color online) (a) Magnetic field dependence of the magnetic torque for $B \| a$. Inset shows the derivatives of the torque curves, indicating the existence of the magnetic transition at $14.5 \mathrm{~T}$. (b), (c) Schematic pictures of the magnetic states of the $\mathrm{Fe}(\mathrm{Pc})(\mathrm{CN})_{2}$ chains. (b) AF correlated state of Fe local moments, and (c) magnetic-field-induced ferromagnetic state.

energy-gap reduction is likely related to the enhancement of $t$ which is associated with the magnetic torque anomaly. Even a small change in the energy gap should cause a drastic change in the conductivity at low temperatures when the carrier number is determined by the Boltzmann factor as in this case.

A theoretical picture of the negative MR for this system is first proposed by Hotta et al. In this picture, the Fe local moment and the $\pi$ electron on the same $\mathrm{Fe}(\mathrm{Pc})(\mathrm{CN})_{2}$ molecule are assumed to form a spin singlet state due to the antiferromagnetic (AF) $\pi$ - $d$ interaction. ${ }^{22}$ This singlet formation effectively reduces $t$, and stabilizes the insulating state. In high fields, the singlet state is broken, so that $t$ is recovered. However, the ferromagnetic $\pi-d$ interaction is predicted by the recent molecular-orbital calculation for the $\mathrm{Fe}(\mathrm{Pc})(\mathrm{CN})_{2}$ molecule, so that the above picture would not be appropriate. ${ }^{23}$

CMR in some perovskite manganites have been explained by the double-exchange-interaction scenario, where the ferromagnetic Hund coupling of the $3 d$ spins on the Mn sites plays an important role. ${ }^{2-4} \mathrm{~A}$ similar scenario would be applicable to the large negative $\mathrm{MR}$ in $\mathrm{TPP}\left[\mathrm{Fe}(\mathrm{Pc})(\mathrm{CN})_{2}\right]_{2}$ [Figs. 4(b) and 4(c)] because the $\pi$ electron and the Fe local moment on the $\mathrm{Fe}(\mathrm{Pc})(\mathrm{CN})_{2}$ site are ferromagnetically coupled. If the Fe local moment at the next site is antiparallel, the hopping of the $\pi$-electron is prohibited by the ferromagnetic $\pi$ - $d$ interaction, so that $t$ is effectively reduced. In high magnetic fields, since all the Fe local moments are polarized in the field direction, $t$ is recovered. Although no experimental evidence of long-range order of the Fe local moments, development of the AF correlation in the $\mathrm{Fe}(\mathrm{Pc})(\mathrm{CN})_{2}$ chain below $25 \mathrm{~K}$ is suggested from the magnetic-susceptibility measurements. ${ }^{11}$ Therefore, some behavior similar to a metamagnetic transition may be observed in the torque at low enough temperatures because of the strong uniaxial anisotropy of the Fe local moment. This picture qualitatively explains the steep change in the MR and the torque around $15 \mathrm{~T}$. The recovery of $t$ in high magnetic fields is also consistent with the magnetic field dependence of the energy gap shown in the inset of Fig. 1. The magnetic transitionlike behaviors observed in MR and magnetic torque 

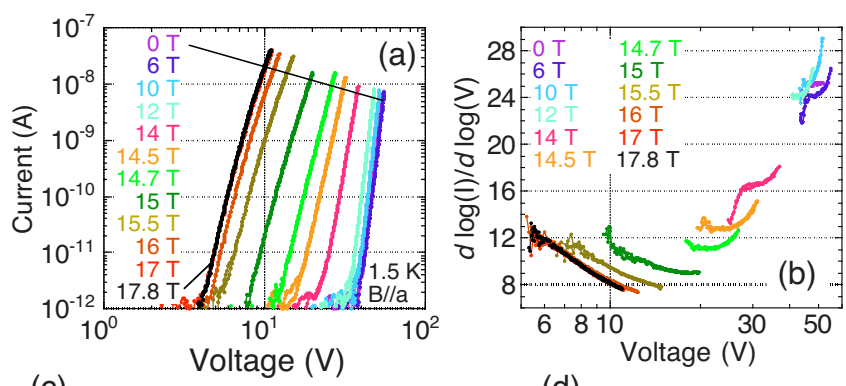

(c)

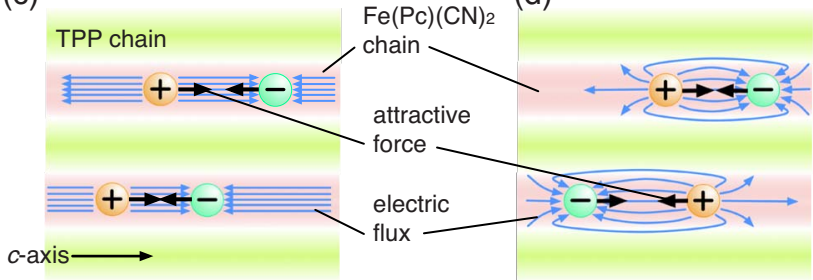

FIG. 5. (Color online) (a) $I-V$ curves at $1.5 \mathrm{~K}$ and (b) their derivative curves. Schematic pictures of electric fluxes between the electron-hole pairs for (c) linear potential case $(B<15$ T: $1 \mathrm{D}$ Coulomb interaction), and (d) square-root potential case $(B>15 \mathrm{~T}$ : quasi-1D Coulomb interaction).

measurements are also observed for various magnetic field directions (data not shown). The transition field shifts to higher field as the magnetic field is tilted from the $a$-axis, and the angular dependence of the transition fields decided from the both measurements are consistent with each other. The detail will be described elsewhere.

Finally, we discuss the change in the functional form of the Coulomb potential by magnetic fields. The magnetic field dependence of the $I-V$ curves and their derivatives at $1.5 \mathrm{~K}$ are shown in Figs. 5(a) and 5(b), respectively. As field increases, the curvature changes from positive to negative around $15 \mathrm{~T}$ [more evident in Fig. 5(b)]. The curvature in the nonlinear region of the $I-V$ characteristics depends on the functional form of the Coulomb potential: $\phi(r) \propto|r|$ for 1D gives the positive curvature as shown in Fig. 2(b), and $\phi(r) \propto \log (r)$ for 2D gives a straight line (power-law behavior). ${ }^{19}$ The form $\phi(r) \propto \sqrt{|r|}$ assumed in the simulation of Fig. 2(e) corresponds to an intermediate case between 1D and 2D. This does not mean that the electrical fluxes are confined in a plane, but simply, the flux density decays with $|r|$ more slowly than the 2D case. Although it is not clear whether only the functional form $\phi(r) \propto \sqrt{|r|}$ is appropriate in high magnetic fields, we could conclude that the electrical fluxes are almost confined in the chains at $0 \mathrm{~T}$ [Fig. 5(c)], but spreads out in the perpendicular directions of the chains in high magnetic fields [Fig. 5(d)]. The electric flux distribution determined by the dielectric constant anisotropy may be related to the anisotropy of $t$ : the dielectric constant (electric polarization) is large along the large $t$ direction because the charges easily move in this direction. In the magnetic-fieldinduced ferromagnetic state, all the $\pi$-electron spins and the Fe local moments are polarized, so that $t$ perpendicular to the chains may be also enhanced, which allows the electric fluxes to spread out somewhat in the perpendicular directions.

\section{CONCLUSIONS}

In conclusion, the quite large $\mathrm{MR}$ in $\mathrm{TPP}\left[\mathrm{Fe}(\mathrm{Pc})(\mathrm{CN})_{2}\right]_{2}$ is interpreted as the energy-gap reduction caused by the magnetic transition like behavior of the Fe local moments in the $\mathrm{Fe}(\mathrm{Pc})(\mathrm{CN})_{2}$ chains, where the strong $\pi-d$ interaction plays a crucial role. The change in the curvature in the nonlinear $I-V$ characteristics shows the dimensionality change in the Coulomb interaction which is affected by the magnetic state of the Fe local moments. The dimensionality and the strength of Coulomb interaction are significantly important for understanding the electronic states in strongly correlated systems, especially in low dimensional systems. Our results show that simple measurements of $I-V$ characteristics can give microscopic information on the dimensionality of the Coulomb interaction.

\section{ACKNOWLEDGMENTS}

We are grateful to N. Hanasaki, M. Matsuda, H. Tajima, C. Hotta, and Y. Otsuka for valuable discussions. This work was partially supported by a Grant-in-Aid for Young Scientists (start-up) (Grant No. 20840044) from the Japan Society for the Promotion of Science. This research was partially supported by a Grant-in-Aid for Scientific Research on Innovative Areas (Grant No. 20110004) from the Ministry of Education, Science, Sports and Culture.

\footnotetext{
*kimata.motoi@nims.go.jp

${ }^{\dagger}$ Also at Graduate School of Pure and Applied Sciences, University of Tsukuba, Tsukuba 305-8577, Japan.

${ }^{1}$ M. N. Baibich, J. M. Broto, A. Fert, F. Nguyen Van Dau, F. Petroff, P. Etienne, G. Creuzet, A. Friederich, and J. Chazelas, Phys. Rev. Lett. 61, 2472 (1988).

${ }^{2}$ Y. Tokura, A. Urushibara, Y. Moritomo, T. Arima, A. Asamitsu, G. Kido, and N. Furukawa, J. Phys. Soc. Jpn. 63, 3931 (1994).

${ }^{3}$ Y. Tomioka, A. Asamitsu, Y. Moritomo, H. Kuwahara, and Y. Tokura, Phys. Rev. Lett. 74, 5108 (1995).

${ }^{4}$ H. Kuwahara, Y. Tomioka, Y. Moritomo, A. Asamitsu, M. Kasai, R. Kumai, and Y. Tokura, Science 272, 80 (1996).
}

${ }^{5}$ S. Uji, H. Shinagawa, T. Terashima, T. Yakabe, Y. Terai, M. Tokumoto, A. Kobayashi, H. Tanaka, and H. Kobayashi, Nature (London) 410, 908 (2001); L. Balicas, J. S. Brooks, K. Storr, S. Uji, M. Tokumoto, H. Tanaka, H. Kobayashi, A. Kobayashi, V. Barzykin, and L. P. Gor'kov, Phys. Rev. Lett. 87, 067002 (2001).

${ }^{6}$ F. Lévy, I. Sheikin, B. Grenier, and A. D. Huxley, Science 309, 1343 (2005).

${ }^{7}$ N. Hanasaki, H. Tajima, M. Matsuda, T. Naito, and T. Inabe, Phys. Rev. B 62, 5839 (2000).

${ }^{8}$ N. Hanasaki, M. Matsuda, H. Tajima, E. Ohmichi, T. Osada, T. Naito, and T. Inabe, J. Phys. Soc. Jpn. 75, 033703 (2006). 
${ }^{9}$ M. M. Matsushita, H. Kawakami, T. Sugawara, and M. Ogata, Phys. Rev. B 77, 195208 (2008).

${ }^{10}$ T. Fujimoto, S. Yasuzuka, K. Yokogawa, H. Yoshino, T. Hayashi, H. Fujiwara, T. Sugimoto, and Keizo Murata, J. Phys. Soc. Jpn. 77, 014704 (2008).

${ }^{11}$ H. Tajima, G. Yoshida, M. Matsuda, K. Nara, K. Kajita, Y. Nishio, N. Hanasaki, T. Naito, and T. Inabe, Phys. Rev. B 78, 064424 (2008).

${ }^{12}$ T. Inabe and H. Tajima, Chem. Rev. (Washington, D.C.) 104, 5503 (2004).

${ }^{13}$ M. Matsuda, T. Naito, T. Inabe, N. Hanasaki, H. Tajima, T. Otsuka, K. Awaga, B. Narymbetov, and H. Kobayashi, J. Mater. Chem. 10, 631 (2000).

${ }^{14}$ N. Hanasaki, M. Matsuda, H. Tajima, T. Naito, and T. Inabe, J. Phys. Soc. Jpn. 72, 3226 (2003).

${ }^{15}$ N. Hanasaki, K. Masuda, K. Kodama, M. Matsuda, H. Tajima, J.
Yamazaki, M. Takigawa, J. Yamaura, E. Ohmichi, T. Osada, T. Naito, and T. Inabe, J. Phys. Soc. Jpn. 75, 104713 (2006).

${ }^{16}$ E. Ohmichi and T. Osada, Rev. Sci. Instrum. 73, 3022 (2002).

${ }^{17}$ H. Seo, C. Hotta, and H. Fukuyama, Chem. Rev. (Washington, D.C.) 104, 5005 (2004).

${ }^{18}$ J. Frenkel, Phys. Rev. 54, 647 (1938).

${ }^{19}$ Y. Takahide, T. Konoike, K. Enomoto, M. Nishimura, T. Terashima, S. Uji, and H. M. Yamamoto, Phys. Rev. Lett. 96, 136602 (2006).

${ }^{20}$ L. V. Keldysh, Phys. Status Solidi A 164, 3 (1997).

${ }^{21}$ H. Mori, S. Tanaka, and T. Mori, Phys. Rev. B 57, 12023 (1998).

${ }^{22}$ C. Hotta, M. Ogata, and H. Fukuyama, Phys. Rev. Lett. 95, 216402 (2005).

${ }^{23}$ D. E. C. Yu, M. Matsuda, H. Tajima, A. Kikuchi, T. Taketsugu, N. Hanasaki, T. Naito, and T. Inabe, J. Mater. Chem. 19, 718 (2009). 